\title{
Fosdenopterin: a First-in-class Synthetic Cyclic Pyranopterin Monophosphate for the Treatment of Molybdenum Cofactor Deficiency Type A
}

\author{
Bernd Schwahn 1,2
}

1. Manchester Centre for Genomic Medicine, St Mary's Hospital, Manchester University NHS Foundation Trust, Health Innovation Manchester Manchester, UK; 2. Division of Evolution \& Genomic Sciences, School of Biological Sciences, Faculty of Biology, Medicine and Health, University of Manchester, Manchester, UK ubstitution of endogenous cyclic pyranopterin monophosphate (CPMP) with fosdenopterin is the first causal treatment for molybdenum cofactor deficiency type A, and can prevent disastrous disability and early death. The development of CPMP substitution therapy started with a unique collaboration between researchers and clinicians, and is being completed in clinical trials sponsored by the pharmaceutical industry. Its safety and efficacy have been demonstrated in a small number of patients over the last decade. The benefit of CPMP substitution is remarkable when started in pre-symptomatic children. The disease-modifying effect of cPMP substitution is less obvious in children whose treatment is started after onset of irreversible brain injury, and CPMP substitution entails a high burden of treatment. A high index of suspicion, easy access to diagnostic tests, and quick supply of fosdenopterin are all crucial to ensure good clinical outcomes. Surveillance of treatment outcomes and further research into alternative modes of drug delivery will aid wider implementation and further development of this novel treatment.

\section{Keywords}

Molybdenum cofactor deficiency, cPMP, fosdenopterin, sulphite, xanthine, urate

Disclosures: Bernd Schwahn was principal investigator for clinical trials sponsored by Alexion Pharma International, Sarl and Origin Biosciences, Inc. He has received travel reimbursement for attendance at a meeting and an unconditional grant to fund an educational meeting about molybdenum cofactor deficiency from Origin Biosciences, Inc.

Review process: Double-blind peer review.

Compliance with ethics: This study involves a review of the literature and did not involve any studies with human or animal subjects performed by any of the authors.

Authorship: The named author meets the International Committee of Medical Journal Editors (ICMJE) criteria for authorship of this manuscript, takes responsibility for the integrity of the work as a whole, and has given final approval for the version to be published.

Access: This article is freely accessible at touchNEUROLOGY.com (c) Touch Medical Media 2021

Received: 2 August 2021

Accepted: 29 September 2021

Published online: 13 December 2021

Citation: touchREVIEWS in Neurology. 2021;17(2):85-91 Corresponding author: Bernd Schwahn, Genomic Medicine, Saint Mary's Hospital, Oxford Road, Manchester, M13 9WL, UK. E: bernd.schwahn@nhs.net

Support: No funding was received for the publication of this article.
Molybdenum cofactor deficiency (MOCD) is an ultra-rare genetic disease that remained untreatable until the recent introduction of cyclic pyranopterin monophosphate (CPMP) substitution. This article aims to comprehensively review the rationale behind cPMP substitution, its effectiveness in clinical scenarios, the development of current treatment protocols, and remaining unmet needs. The clinical manifestations of MoCD, its pathology, current concepts of disease mechanisms and available biomarkers are summarized to help understand achievable outcomes and limitations of cPMP substitution.

\section{Synthesis of cyclic pyranopterin monophosphate and molybdoenzymes in humans}

Humans require molybdenum (Mo) as a catalyst for four oxidoreductases. The cell biology of molybdenum and the synthesis of the molybdenum cofactor (MOCo) have been reviewed in detail elsewhere. ${ }^{1,2,3}$ In short, the synthesis of mature MoCo requires four steps, involving substrate transport across membranes, and enzyme complexes in mitochondria and the cytosol (Figure 1), which are encoded by four genes with multiple gene products (Figure 2).

CPMP was identified in 1993 as Precursor Z, the first and most stable intermediate in MoCo synthesis. ${ }^{4}$ When it was confirmed that Precursor $Z$ exists in the cyclo-pyrano form, the alternative name CPMP was proposed. ${ }^{5}$ CPMP is a tricyclic pteridine molecule that is synthesised in the mitochondrial matrix directly from guanosine- $5^{\prime}$-triphosphate (GTP) by two enzymes, molybdenum cofactor synthesis (MOCS)1A and MOCS1B, which are alternative products of the MOCS1 gene. ${ }^{6}$ CPMP is exported into the cytosol where two sulphur atoms are incorporated, producing molybdopterin (MPT). This step is facilitated by the MPT synthase complex, comprising MOCS2A and MOCS2B, alternative products of the MOCS2 gene.? MOCS3, encoded by the MOCS3 gene, is required to regenerate MPT synthase. ${ }^{8}$ Gephyrin, encoded by the GPHN gene, facilitates the adenylation of MPT and subsequent insertion of molybdate with cleavage of adenylate to form the mature MoCo. ${ }^{9}$

CPMP, MPT and MoCo are all sensitive to oxidative inactivation. MPT and MoCo have a very short biological half-life in oxidative environments. ${ }^{10} \mathrm{MoCo}$ is protected against oxidation to Form $\mathrm{B}$ and enzymatic degradation to urothione by binding to specific binding proteins ${ }^{11}$ or by incorporation into one of the apoproteins of the four known human molybdoenzymes..$^{12,13}$ The mature MoCo is directly incorporated as a prosthetic group into the nascent apoproteins of sulphite oxidase and 
Figure 1: Simplified overview of the compartmentalization of molybdenum cofactor synthesis and of relevant molybdoenzymes and their metabolites

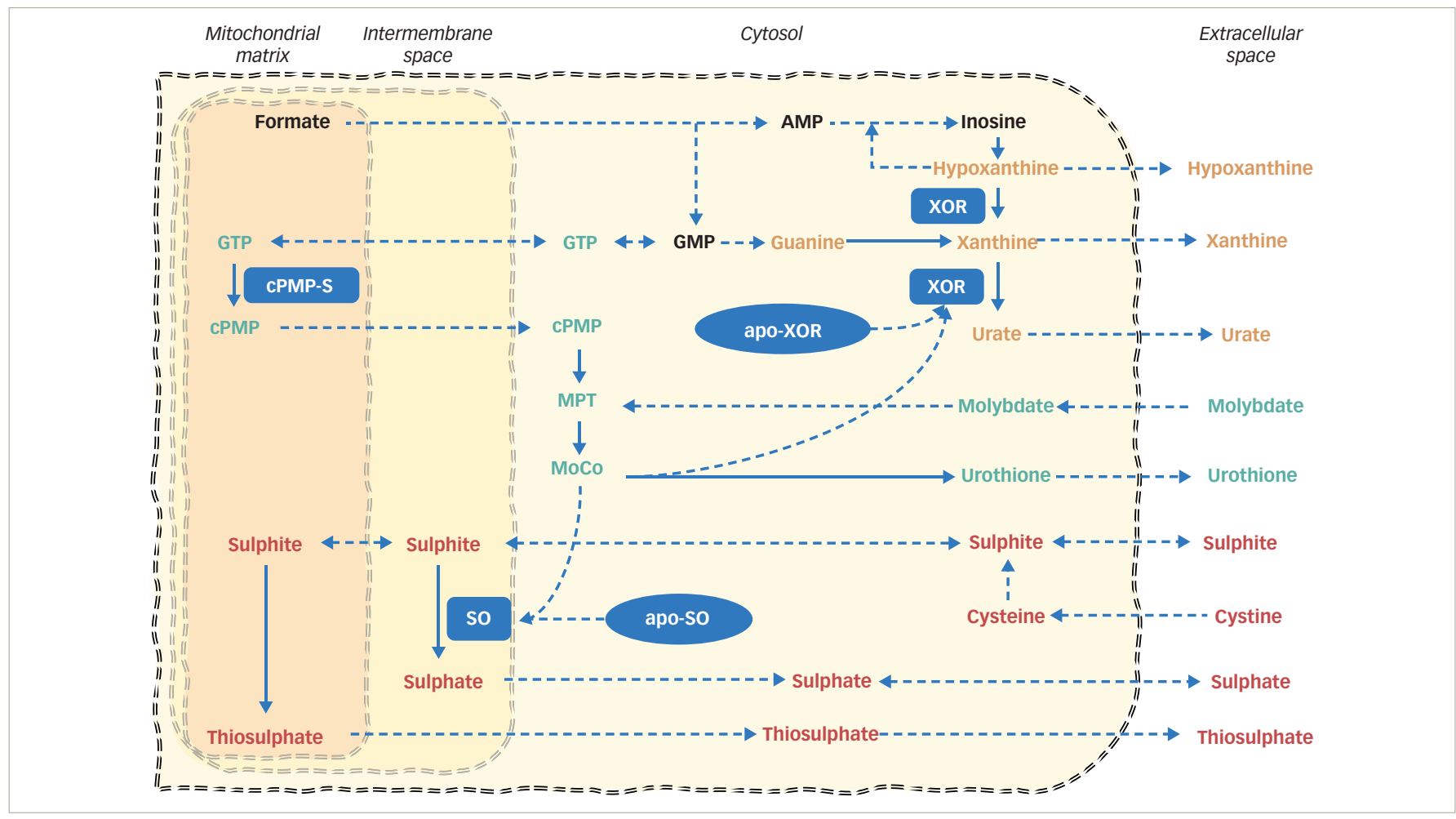

CPMP is synthesized in the mitochondrial matrix from GTP by two enzymes, MOCS1A and MOCS1B, alternative products of the MOCS1 gene6 that act as a CPMP synthase. CPMP is exported into the cytosol where two sulphur atoms are incorporated, producing MPT. This step is facilitated by the MPT synthase complex, comprising MOCS2A and MOCS2B, alternative products of the MOCS2 gene. ${ }^{7}$ MOCS3 is required to regenerate MPT synthase. ${ }^{8}$ MPT is then adenylated by gephyrin to allow molybdate to be incorporated. ${ }^{9}$ The resulting molybdenum cofactor is unstable but can escape rapid degradation to urothione by binding to molybdenum cofactor binding proteins and by being incorporated into cytosolic apoproteins including SO and XOR. ${ }^{12,13}$ SO converts highly reactive sulphite into inert sulphate. XOR helps remove excess purine bases by converting hypoxanthine to xanthine and, eventually, urate. Sulphite is derived from the metabolism of sulphur amino acids, especially from cysteine degrading in liver ${ }^{71}$ As a small molecule, sulphite moves easily between body compartments. Accumulating sulphite in mitochondria can be converted to thiosulphate, which is excreted in urine.

Solid blue lines represent direct reactions and dashed blue lines represent metabolic pathways and transport processes. Molecule colours reflect groups of related metabolites red, sulphur metabolism; green, molybdenum metabolism; yellow, purine metabolism.

$A M P=$ adenosine monophosphate; $C P M P=$ cyclic pyranopterin monophosphate; $c P M P-S=$ cyclic pyranopterin monophosphate synthase; GMP = guanosine monophosphate; GTP = guanosine triphosphate; $M P T=$ molybdopterin; $S O=$ sulphite oxidase; $X O R=$ xanthine oxidoreductase.

of the mitochondrial amidoxime reducing component. The incorporation of MoCo is effectively required for mitochondrial import and retention of sulphite oxidase (SO)..$^{2}$ In contrast, MoCo must be modified by MoCo sulphurase before it can bind as cofactor to the apoproteins of xanthine oxidoreductase (XOR) or aldehyde oxidase (AO). ${ }^{14}$

\section{Clinical presentation of molybdenum cofactor deficiency}

Defects in MoCo synthesis lead to deficiencies of all molybdoenzymes, except for defects in MoCo sulphurase, which only affect XOR and $\mathrm{AO}$ (Figure 2). By far, the most relevant consequence of MoCD is the resulting lack of SO activity. Isolated sulphite oxidase deficiency (ISOD), due to genetic variants in the SUOX gene, causes a distinctive infantile neurological syndrome that was first described in 1967.15,16 MoCD was first described in $1978 .{ }^{17}$ Clinically, ISOD and MoCD present with the same symptoms and complications caused by sulphite accumulation, except those patients with MoCD can, in addition, develop nephrolithiasis due to impaired XOR activity. ${ }^{18}$

MOCD and ISOD are ultra-rare diseases, with published reports of fewer than 200 patients with MOCD ${ }^{19-21}$ and fewer than 100 with ISOD. ${ }^{22}$ Biallelic pathogenic variants in MOCS1 impair the production of CPMP and are classified as MoCD type A (MoCD-A), which is the most commonly diagnosed subtype of MoCD. Most other cases are MoCD type B (MoCD-B), caused by MOCS2 variants ${ }^{23}$ or, rarely, MOCS3 mutations. ${ }^{24,25}$
Only a few cases of MoCD type C (MoCD-C) (caused by GPHN mutations) have been found. ${ }^{26}$ There are no published data on the incidence of MoCD available. From the author's own unpublished observation, the prevalence at birth is at least 1 in 200,000 newborns in the UK.

Pre-natal brain abnormalities have been reported in a small number of infants with MoCD, including subcortical cysts, dysgenesis of the corpus callosum, ${ }^{27}$ as well as enlarged lateral ventricles and, more commonly, an enlarged cisterna magna and cerebellar hypoplasia.28,29 These abnormalities indicate that early brain injury occurs in some cases from mid-gestation onwards. ${ }^{29}$ Mild brain oedema has been identified in some foetuses from 36 weeks of gestation, ${ }^{28}$ leading to consideration of early delivery for affected infants. ${ }^{28,30}$ Protection from accumulating sulphite through trans-placental maternal clearance ${ }^{30,31}$ ceases at birth, resulting in fulminant encephalopathy in infants with severe MoCD, which manifests within hours to days after delivery. Clinical signs are feeding difficulties, irritability, exaggerated startle reactions, decreased consciousness, apnoea, seizures, axial hypotonia and limb hypertonia.. ${ }^{18}$ Newborns can manifest with moderate metabolic acidosis and increased lactate, as well as with hypoglycaemia. Other routinely performed biochemical investigations are typically normal. Brain magnetic resonance imaging (MRI) at this early stage of disease reveals generalized oedema and widespread restricted diffusion, indicating neuronal damage..$^{20}$ The presentation is similar to that of hypoxic brain injury, for which it may be mistaken. 
Figure 2: Molybdenum cofactor synthesis and biochemical consequences of molybdenum cofactor deficiency for relevant biomarkers

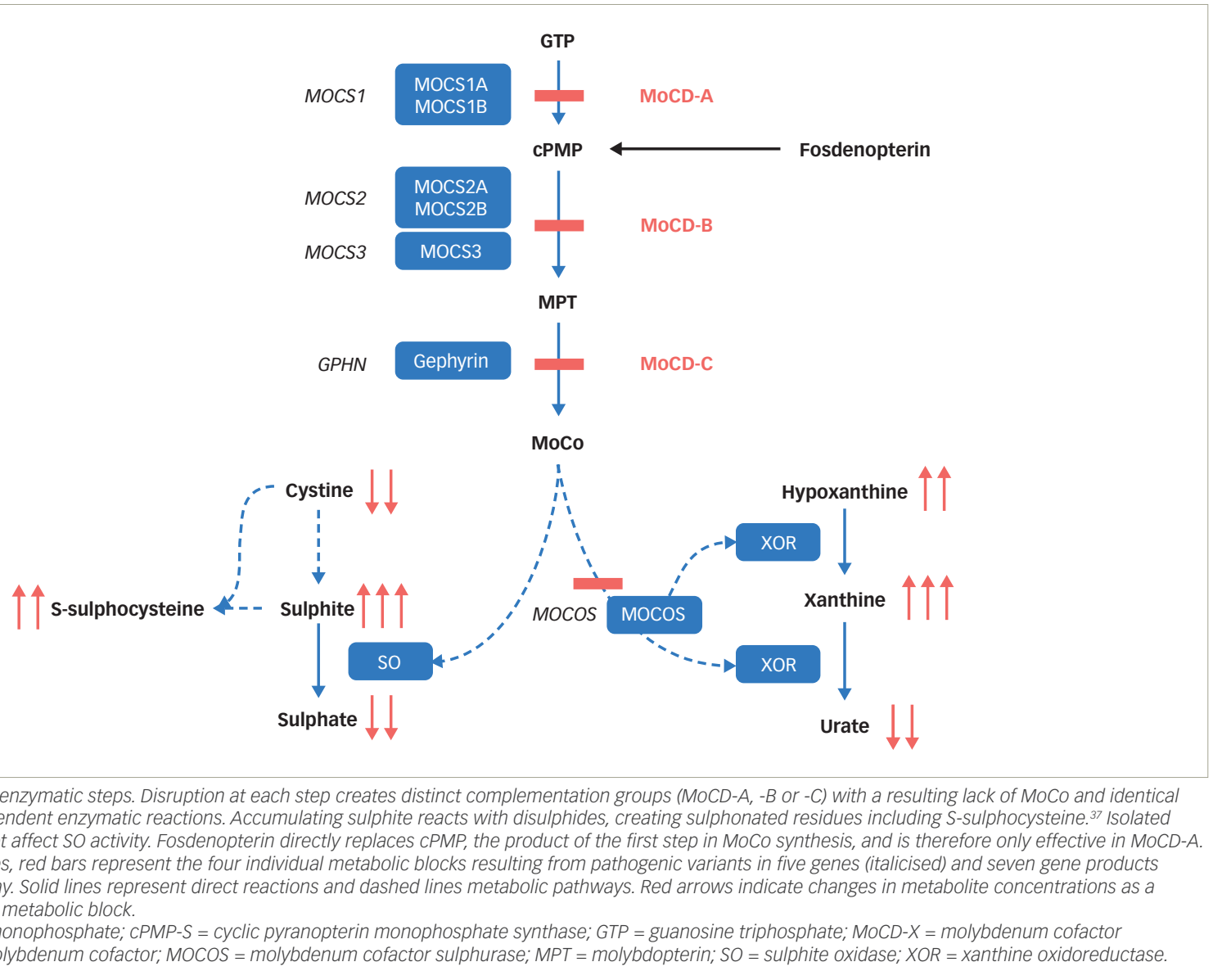

After the neonatal period, surviving infants develop a relatively uniform phenotype. They continue to be irritable, have myoclonic and generalized seizures, and develop severe spastic cerebral palsy with intermittent dystonic crises. They typically have profound developmental delay and their brain is atrophic with acquired porencephaly and microcephaly. Children are at high risk of secondary complications from seizures, aspiration, and lower respiratory tract infections. Older children display marfanoid features, including lens dislocation, and a few cases of urolithiasis due to xanthinuria have been reported..$^{18,32}$ Their reported median age at death is between 2.4 years ${ }^{21}$ and 3.0 years, ${ }^{19}$ and is dependent on the severity of illness but also on the extent of medical care provided.

Attenuated disease manifestations are being increasingly reported and likely represent a milder biochemical defect. Symptom onset may occur during infancy or childhood and can be of insidious or sudden onset, with dystonia, spasticity, and varying degrees of developmental delay. ${ }^{33,34}$ There is a genotype-phenotype correlation; a few MOCS1 and MOCS2 variants are associated with later disease onset and milder clinical manifestations, ${ }^{21,35}$ including less pronounced biochemical abnormalities. There is, however, no overlap between healthy individuals and those with untreated MoCD. ${ }^{21}$

\section{Biochemical derangement in molybdenum cofactor deficiency and clinically relevant biomarkers}

Most known cases of MoCD are of neonatal onset and are associated with severe biochemical disturbance and disease, including early acute encephalopathy and seizures. MoCD-A and MoCD-B largely overlap in their clinical and biochemical features and can only be distinguished by genetic assessment or measuring urinary cPMP concentrations in a research laboratory. 26,36

SO converts highly reactive sulphite into inert sulphate (Figure 2) ${ }^{15}$ SO deficiency leads to sulphite accumulating, resulting in a multitude of secondary biochemical abnormalities. As a small molecule, sulphite readily moves between body compartments and is found in increased concentrations in all body fluids. ${ }^{18}$ Increased sulphite in urine can be easily detected using sulphite test strips; however, these are not licensed for medical use and can yield false positive and false negative results. ${ }^{18,26}$ In mitochondria, sulphite can be converted to thiosulphate, which is excreted in urine. Sulphite readily reacts with disulphides, including with free L-cystine, yielding S-sulphocysteine (SSC), which is also renally excreted. ${ }^{37}$ Thiosulphate and SSC are reliable and stable markers of sulphite accumulation that rise very quickly after birth and can be measured in blood or urine in specialized laboratories. SSC concentrations show great interindividual but little intraindividual variability over time. ${ }^{21}$

XOR is involved in removing excess purine bases by converting hypoxanthine to xanthine and eventually to urate (Figure 2). The lack of XOR activity causes xanthine and hypoxanthine to accumulate, as well as greatly reducing urate production. Plasma urate is in equilibrium with the maternal circulation during pregnancy, and plasma concentrations in neonates with MOCD can remain within the normal range during the first few days of life until renal clearance has removed all maternal urate from their circulation. ${ }^{18}$ Plasma urate is normal in ISOD and can be decreased 
but present in milder MoCD. ${ }^{21}$ Plasma or urinary purine metabolites are a more sensitive marker of compromised XOR activity and can be measured in specialized metabolic laboratories. ${ }^{18}$

\section{Current hypotheses of pathomechanisms in molybdenum cofactor deficiency}

MoCD is a primarily neurological disease affecting neurons in the central and peripheral nervous system. The disease course suggests acute neuronal energetic failure and research has shown that mitochondria energy metabolism is directly impaired by accumulating sulphite. ${ }^{38}$ Sulphite increases oxidative stress and reduces adenosine triphosphate (ATP) synthesis by directly inhibiting glutamate hydrogenase in mitochondria that respire on glutamate hydrogenase. ${ }^{39}$ Sulphite can disrupt mitochondrial integrity and function, ${ }^{40}$ and mitochondrial respiration was found to be impaired in a MOCS1 defective cell line ${ }^{41}$ and in cells of SO-deficient mice. ${ }^{22}$ In addition, a specific pathomechanism has been proposed that can explain the apparent peracute neuronal failure after birth: SSC is a strong N-methyl-D-aspartate (NMDA) receptor agonist, ${ }^{43,44}$ and leads to excitotoxic neuronal cell death and seizures. ${ }^{45-47}$ It is therefore likely that, in MoCD, the encephalopathic crisis after birth is caused by an excitotoxic storm that leads to widespread neuronal necrosis.

Cleavage of protein disulphide bridges by sulphite alters the tertiary structure and function of enzymes and structural proteins. ${ }^{37}$ Cleavage of such structural proteins disrupts connective tissue, causing marfanoid features and lens dislocation. ${ }^{18}$

Direct inhibition of the enzyme alpha-amino adipic semialdehyde (AASA) by sulphite has been demonstrated and leads to a secondary deficiency of bioavailable pyridoxal 5'-phosphate, which may contribute to hyperexcitability and seizure activity. ${ }^{48,49}$

\section{Development of cyclic pyranopterin monophosphate treatment for molybdenum cofactor deficiency type A}

\section{Preclinical work}

Until recently, there was no causal treatment for MoCD. Affected children have benefitted from symptomatic and supportive treatment. Sulphite accumulation can be reduced with dietary restriction of sulphur-containing amino acids, ${ }^{50}$ but any attempts to modify the course of disease in severe MoCD, for example with administration of ammonium molybdate, sulphate, thiamine, D-penicillamine, mesna and tetrahydrobiopterin, were unsuccessful. Supplementation with pyridoxine has been suggested in milder cases of MoCD.51

Replacing the lacking MoCo has been tried for decades. Directly replacing isolated MoCo or MPT is hampered by their lability in aerobic environments. However, co-cultivation of cells from different MOCD patients could restore the concentration of MPT and activity of molybdoenzymes in some cell lines, suggesting the presence of a diffusible intermediate in cofactor synthesis and the existence of a stable precursor to MPT.52 Although this precursor was identified in $1993,{ }^{4}$ it took another 10 years to establish its exact structure. ${ }^{5}$ Creating a transgenic MOCS1 defective mouse model, ${ }^{53}$ and the recombinant overexpression of CPMP-producing enzymes in Escherichia coli eventually allowed testing the previously proposed substrate replacement with CPMP in vivo. E. coli-derived and purified CPMP (rCPMP) given to MOCS1 -/- mice within 5 days after birth and then every 3 days per transabdominal intrahepatic injection ensured survival and normalisation of xanthine and SSC. Liver MPT levels reconstituted to maximally $16 \%$ of wild type on the day of injection. SO activity reached a maximum of $26 \%$ and XOR reached around $40 \%$ of wild-type activity on day 1 after injection. Stopping the treatment led to behavioural deterioration after 6 days of withdrawal. ${ }^{54}$

\section{First-in-human use and administration of cyclic pyranopterin monophosphate on named patient basis}

The administration of rCPMP to humans with MoCD started in 2008 on a named patient basis as an experimental treatment and according to a strict prospective observational protocol designed by Orphatec Pharmaceuticals $\mathrm{GmbH}$ (later renamed Colbourne Pharmaceuticals $\mathrm{GmbH}$ ), a spin-off from the original research group. ${ }^{55}$ The first child with MoCD-A was 36 days old when treatment started, and responded within a few days with normalized biomarkers and clinical improvement. However, the sequelae of post-natal neuronal injury could not be averted, and the child later showed typical signs of severe cerebral palsy. ${ }^{36}$ The second infant with MoCD-A was treated in 2009 under similar circumstances but treatment was started earlier, at the age of 7 days, and prior to developing seizures. This child showed normalized biomarkers and normal early development. ${ }^{56} \mathrm{~A}$ third child was treated in 2010 from the age of 5 days. This child was already severely encephalopathic at the start of treatment and, while biomarkers improved as expected, the child developed typical sequelae of brain injury and severe cerebral palsy. ${ }^{56,57} \mathrm{~A}$ further two neonates were treated in $2010^{58}$ and 2011, within hours after birth, and both showed moderate developmental delay in childhood. ${ }^{28}$ No adverse reactions to treatment were observed under continued CPMP administration for these and a few other children. ${ }^{59}$

In September 2010, prompted by these encouraging results, the European Commission granted orphan designation for CPMP for the treatment of MoCD-A to Orphatec Pharmaceuticals GmbH..$^{60}$ In 2011, Alexion Pharma International Sarl acquired the CPMP programme and started chemically synthesizing CPMP. The synthetically derived CPMP, fosdenopterin, has identical properties to the naturally occurring rcPMP.61 In March 2013, the European Medicines Agency transferred sponsorship to Alexion Europe SAS, who also received breakthrough therapy designation from the US Food and Drug Administration (FDA) in the same year. Origin Biosciences Inc acquired the late stage CPMP therapy programme in 2018 and, in February 2021, the FDA approved fosdenopterin for the treatment of MoCD-A in the USA. ${ }^{62}$

\section{Clinical trials and treatment experience with cyclic pyranopterin monophosphate}

The circumstances and outcomes of the first cohort of 16 infants treated with recombinant CPMP on a named patient basis and following a prospective monitoring protocol were published in 2015.59 Five of those infants were siblings of previous index cases, including one child with MoCD-B. Eleven others were diagnosed based on their symptoms after birth and, of those, seven were affected with MOCD-A and four with MoCD-B. Treatment in all five infants with MoCD-B was terminated after lack of response to CPMP substitution. Treatment was also discontinued in five of the 11 infants with MoCD-A who showed a biochemical response but whose disease had progressed to an extent that CPMP substitution was deemed futile by the treating physicians and parents.

From 2011, Alexion Pharma International Sarl had designed and sponsored a series of international clinical trial protocols to develop cPMP therapy (Table 1), ${ }^{63.66}$ which were later continued by Origin Biosciences Inc. Studies ALX-MCD-501 (NCT01640717) ${ }^{63}$ and ALXN1101-MCD-101 (NCT01894165)64 have been completed. The trials ORGN001-MCD-201 (NCT02047461, EudraCT 2013-002701-56) ${ }^{65}$ and ORGN001-MCD-202 (NCT02629393, EudraCT 2013-002702-30) ${ }^{66}$ are ongoing. Data from these trials have not 
Table 1: Clinical trials within the fosdenopterin development programme

\begin{tabular}{|c|c|c|c|c|c|}
\hline Name & $\begin{array}{l}\text { Identifiers ClinicalTrials.gov } \\
\text { EudraCT }\end{array}$ & Study type & $\begin{array}{l}\text { Individuals } \\
\text { treated }\end{array}$ & $\begin{array}{l}\text { cPMP doses } \\
\text { explored }\end{array}$ & Reference \\
\hline ALX-MCD-501 & NCT01640717 & $\begin{array}{l}\text { Retrospective, observational, non-interventional data } \\
\text { collection study for patients with MoCD who have been } \\
\text { previously treated with recombinant cyclic pyranopterin } \\
\text { monophosphate (rcPMP) }\end{array}$ & $\begin{array}{l}10 \text { infants with } \\
\text { MoCD-A } \\
5 \text { infants with } \\
\text { MoCD-B }\end{array}$ & $80-320 \mu g / k g$ & 63 \\
\hline ALXN1101-MCD-101 & NCT01894165 & $\begin{array}{l}\text { Phase 1, randomized, blinded, placebo-controlled, single- } \\
\text { dose, sequential-cohort, dose-escalation study to evaluate } \\
\text { the safety, tolerability and pharmacokinetics of ALXN1101 } \\
\text { in healthy adult subjects }\end{array}$ & $\begin{array}{l}18 \text { healthy adult } \\
\text { volunteers }\end{array}$ & $75-680 \mu g / k g$ & 64 \\
\hline $\begin{array}{l}\text { ORGN001-MCD-201 } \\
\text { formerly } \\
\text { ALXN1101-MCD-201 }\end{array}$ & $\begin{array}{l}\text { NCT02047461 } \\
\text { EudraCT 2013-002701-56 }\end{array}$ & $\begin{array}{l}\text { Phase 2, multicenter, multinational, open-label, dose- } \\
\text { escalation study to evaluate the safety and efficacy of } \\
\text { ALXN1101 in pediatric patients with MoCD type A currently } \\
\text { treated with recombinant Escherichia Coli-derived cyclic } \\
\text { pyranopterin monophosphate (rCPMP) }\end{array}$ & $\begin{array}{l}8 \text { children with } \\
\text { MoCD-A (6 enrolled } \\
\text { from } \\
\text { ALX-MCD-501) }\end{array}$ & $180-900 \mu g / k g$ & 65 \\
\hline $\begin{array}{l}\text { ORGN001-MCD-202 } \\
\text { formerly } \\
\text { ALXN1101-MCD-202 }\end{array}$ & $\begin{array}{l}\text { NCT02629393 } \\
\text { EudraCT 2013-002702-30 }\end{array}$ & $\begin{array}{l}\text { Phase } 2 / 3 \text {, multicenter, multinational, open label study } \\
\text { to evaluate the efficacy and safety of ORGN001 (formerly } \\
\text { ALXN1101) in neonates, infants, and children with MoCD } \\
\text { type A }\end{array}$ & $\begin{array}{l}1 \text { infant with } \\
\text { MoCD-A }\end{array}$ & $400-980 \mu \mathrm{g} / \mathrm{kg}$ & 66 \\
\hline
\end{tabular}

$C P M P=$ cyclic pyranopterin monophosphate; $M O C D-A / B=$ molybdenum cofactor deficiency type $A / B ; r c P M P=$ recombinant cyclic pyranopterin monophosphate

yet been made publicly available, apart from excerpts and summaries contained in the prescribing information for fosdenopterin hydrobromide dihydrate (Nulibry ${ }^{\top M}$, Origin Biosciences, Boston, MA, USA) ${ }^{67}$ and a preliminary poster presentation at a scientific meeting. ${ }^{68}$

Six patients from the original cohort and two additional patients who were treated later remained on rCPMP treatment until they were switched to fosdenopterin. This switch was monitored under clinical trial protocol ORGN001-MCD-201, which also included a dose escalation exercise with pharmacokinetic and pharmacodynamic assessments. ${ }^{65}$ Another patient was started on fosdenopterin under the trial protocol ORGN001-MCD-202, ${ }^{66}$ bringing the total of long-term treated patients to nine. Survival of all infants treated with CPMP was improved compared to matched untreated controls. ${ }^{67}$

\section{Dosage of cyclic pyranopterin monophosphate}

Intravenously administered CPMP has an elimination half-life of 1.21.7 hours, with a proportion of renal clearance of around $40 \% .{ }^{67} \mathrm{~A}$ large proportion of CPMP is oxidized non-enzymatically to compound $\mathrm{Z}$, and a smaller proportion is converted to MPT and eventually to MoCo. Some mature MoCo is incorporated into molybdoenzymes and some degrades to Form B or urothione, which are lost in urine. Estimated daily molybdenum requirements for healthy adults are $25 \mu g .{ }^{69}$ The European Society for Paediatric Gastroenterology Hepatology and Nutrition and The European Society for Clinical Nutrition and Metabolism recommend $0.25 \mu \mathrm{g} / \mathrm{kg} /$ day of molybdenum in term infants and children receiving long-term parenteral nutrition. ${ }^{70}$ Assuming this is sufficient to produce adequate amounts of mature MoCo, equivalent CPMP requirements would be around $100 \mu \mathrm{g} /$ day in adults and $1-2 \mu \mathrm{g} / \mathrm{kg} /$ day in infants. Pharmacological dosing requirements of the precursor CPMP are dependent on mode and frequency of administration and are likely higher when CPMP is given as intermittent intravenous boluses. Schwarz et al. suggested an intravenous treatment dose for cPMP of $100 \mu \mathrm{g} / \mathrm{kg}$ twice weekly, based on their original mouse experimentation and the observed biological half-life of holo-molybdoenzymes, ${ }^{54}$ which is a highly relevant parameter to consider when establishing dosing intervals.
The first few patients were started on a dose of $80 \mu \mathrm{g} / \mathrm{kg}$ CPMP once daily, administered in staggered short intravenous infusions, and the dose was sequentially escalated over $2-3$ months to $240 \mu \mathrm{g} / \mathrm{kg}$ once daily. ${ }^{56}$ Later patients were started on a dose of $240 \mu \mathrm{g} / \mathrm{kg} /$ day. ${ }^{59}$ The licensed starting dose for fosdenopterin is $400 \mu \mathrm{g} / \mathrm{kg}$ in pre-term infants and $550 \mathrm{\mu g} / \mathrm{kg}$ in term infants, both escalating to $900 \mu \mathrm{g} / \mathrm{kg}$ once daily from month 3 of treatment and from the start of treatment for any children over the age of 1 year. ${ }^{67} \mathrm{CPMP}$ substitution in the doses used so far can restore normal concentrations of biomarkers. ${ }^{59}$ Based on studies of the mouse model of MoCD-A, it is likely that the current dosage is not sufficient to restore full enzymatic activity. ${ }^{54}$ This may, however, not be required to prevent harmful sulphite or xanthine accumulation.

\section{Safety of cyclic pyranopterin monophosphate}

The safety of CPMP has been demonstrated since 2008 with daily administration of rcPMP over approximately 3 years in eight patients, and of fosdenopterin hydrobromide dihydrate over a median duration of 4.3 years in nine patients. No immune-mediated infusion-associated reactions or direct toxic effects have been observed. Adverse events reported during clinical trials related to intercurrent illnesses of childhood and complications associated with central venous access. Daily intravenous administration normally requires a surgically placed, tunnelled intravenous line, which incurs a risk for typical complications, such as exit site infection, septicaemia, or catheter blockage. One infant died from necrotising enterocolitis while treated with CPMP. ${ }^{67}$

Animal studies have identified a potential risk of ultraviolet phototoxicity in patients receiving high doses of fosdenopterin. ${ }^{67}$ Patients on CPMP substitution therapy should avoid or minimize exposure to sunlight or artificial ultraviolet light. The number of patients exposed to fosdenopterin is still very small and further safety surveillance is required to determine the relevance of phototoxicity in clinical practice.

\section{Biochemical efficacy of cyclic pyranopterin monophosphate}

Available data demonstrate good and sustained biochemical efficacy with any of the previously used dosing regimens. ${ }^{59}$ In MoCD-A, a decrease 
Figure 3: Chemical structure of fosdenopterin (cyclic pyranopterin monophosphate $)^{72}$

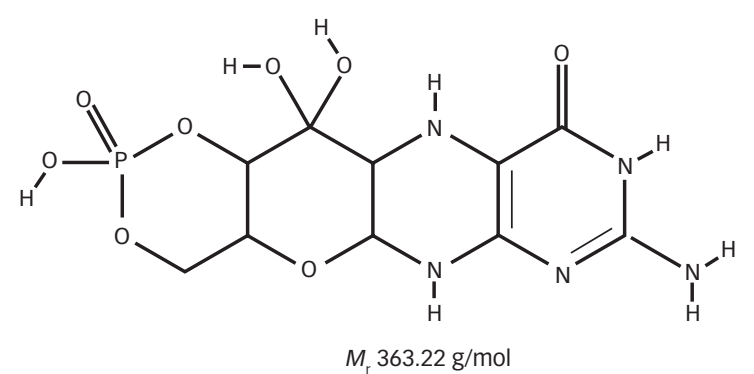

$M=$ molar mass.

Source: https://pubchem.ncbi.n/m.nih.gov/compound/135894389. 2D-Structure

in sulphite, SSC, xanthine and hypoxanthine is expected within 24 hours and an increase in urate in body fluids within 24-48 hours of initiating treatment. Previously grossly abnormal biomarkers remain close to or within the reference range under treatment. ${ }^{59}$ There has been no tachyphylaxis reported in any of the long-term treated patients for over 10 years. Pharmacodynamic data from the clinical trials have not been published, and the optimum dose and administration intervals have not been robustly established yet.

\section{Long-term clinical outcomes of children treated with cyclic pyranopterin monophosphate}

The clinical outcomes of treated patients have been variable. At first look, this may be surprising, given the uniformly good biochemical response to CPMP substitution in patients with MoCD-A. The clinical outcome, however, does not solely depend on the biochemical response of the patient. The neurological outcome of treated infants correlates closely with the state of consciousness at initiation of treatment, limiting relevant developmental progress to those who were treated prior to having entered a comatose state. ${ }^{59}$ Correcting the biochemical abnormality in MoCD-A is expected to protect injured and intact neurons, but cannot reverse any preceding neuronal necrosis. This implies that the neurological outcome of patients mainly depends on the extent of initial brain injury sustained prior to intervention.

A few children with severe MoCD-A have been treated prior to experiencing seizures or becoming comatose and some have been treated immediately after birth. While these patients have not developed cystic encephalomalacia, brain atrophy with secondary microcephaly, or cerebral palsy, and some have never experienced any seizures, sequelae such as speech delay, mild learning difficulties and mild central muscular hypotonia have been observed. ${ }^{28,59}$ This indicates an incomplete treatment effect, either due to subclinical pre- or immediately post-natal brain injury or due to an ongoing disease process that is not reflected in the concentration of currently used plasma and urine biomarkers.

Children whose treatment started after having suffered significant brain injury have continued to have seizures, frequent myoclonus and severely impaired motor and cognitive function, as well as the typical neuroradiological sequelae of MoCD. However, with ongoing CPMP treatment, their survival is improved over a comparable untreated group ${ }^{21,67}$ and their risk of nephrolithiasis due to xanthinuria is abolished due to a normalisation of urinary xanthine concentrations. It is difficult to quantify other presumed benefits of treatment, such as reduced irritability and an improvement in their families' quality of life.
Children with milder MOCD-A and late symptom onset are likely to benefit from CPMP substitution, which may prevent episodes of acute neurological deterioration and improve cognitive developmental progress.

\section{Practical considerations and unmet needs}

Fosdenopterin is supplied as a sterile, preservative-free, lyophilized powder in a single-dose, clear glass vial for reconstitution. Each vial contains $9.5 \mathrm{mg}$ fosdenopterin (equivalent to $12.5 \mathrm{mg}$ fosdenopterin hydrobromide as a dihydrate). ${ }^{67}$ It should be noted that the molar mass of CPMP is $363.22 \mathrm{~g} / \mathrm{mol}$ (Figure 3 ) and that of fosdenopterin monobromide dihydrate is $480.16 \mathrm{~g} / \mathrm{mol}$. After reconstitution, the final concentration is $9.5 \mathrm{mg}$ fosdenopterin in $5.0 \mathrm{~mL}(1.9 \mathrm{mg} / \mathrm{mL})$ and the dose is administered undiluted through a $0.2 \mu \mathrm{m}$ filter as a short intravenous infusion, at a rate of $1.5 \mathrm{~mL} / \mathrm{min}$. Fosdenopterin must be stored frozen between $-10^{\circ} \mathrm{C}$ and $-25^{\circ} \mathrm{C}$ and used within 4 hours after reconstitution.

From the author's personal observation, the requirement for daily intravenous administration puts a significant burden on carers of affected children who administer treatment at home after the initial hospitalization. This requires resources in terms of healthcare provision, space at home and time of carers, and it limits the freedom of movement for the families. While all this can and has been overcome successfully in several cases, more research into dosing intervals and alternative modes of application of fosdenopterin is required to make this treatment more widely available and reduce the risk-benefit ratio.

Timely diagnosis of affected individuals is the greatest challenge. Severely affected neonates may become symptomatic within hours to days after birth, and irreversible brain injury occurs rapidly.58,59 The initial presentation, especially in late-onset cases, is unspecific, and reliable testing for sulphite accumulation is not always readily available. ${ }^{33}$ However, the option of treating children on suspicion of MoCD and prior to diagnostic confirmation is within the licensed application of fosdenopterin. Ideally, a validated bedside test that can detect elevated sulphite and xanthine should be developed to allow timely diagnosis and treatment. Further delays may be incurred if fosdenopterin must be transported to the patient.

While fosdenopterin is an excellent treatment for MoCD-A, other strategies are required to provide treatments for the closely related MOCD-B and MOCD-C, as well as for ISOD.

The following recommendations are based on the author's personal experience. Treatment with cPMP should include close clinical surveillance and initial frequent monitoring of biomarkers, such as xanthine, urate, sulphite and SSC to establish a biochemical response, especially if CPMP substitution is started prior to genetic diagnosis. Measurements of SSC and purines in urine or plasma may not always be available in a timely fashion. Commercially available sulphite dip sticks are not licensed for medical use and, therefore, are not generally accepted for monitoring purposes. Plasma urate is a more commonly used assay, and results are expected to be available in most neonatal and paediatric units within 24 hours. An increase in plasma urate within 2 days of CPMP substitution indicates a treatment response.

\section{Conclusion}

CPMP substitution is a safe and effective causal treatment for MoCD-A that can prevent disastrous disability and early death. The benefits for the child's development are remarkable when treatment is started 
in pre-symptomatic children and justify the burden of treatment. The disease-modifying benefit of fosdenopterin treatment is less clear in children whose treatment is started after they have suffered irreversible neuronal injury.

A high index of suspicion and easy access to diagnostic tests and the drug are crucial to ensure good clinical outcomes. Given its good safety profile, early empirical fosdenopterin treatment of encephalopathic infants with a possible diagnosis of MoCD should be considered.

The requirement for frozen storage and daily intravenous administration of fosdenopterin is challenging in day-to-day practice. Surveillance of treatment outcomes and further research into alternative modes of drug delivery will aid the wider implementation of this novel treatment. $\square$
1. Leimkühler S, Wuebbens MM, Rajagopalan $\mathrm{KV}$. The history of the discovery of the molybdenum cofactor and novel aspects of its biosynthesis in bacteria. Coord Chem Rev. 2011;255:1129-44.

2. Mendel RR. The molybdenum cofactor. $J$ Biol Chem.

2013;288:13165-72

3. Tejada-Jimenez M, Chamizo-Ampudia A, Calatrava V, et al. From the eukaryotic molybdenum cofactor biosynthesis to the moonlighting enzyme mARC. Molecules. 2018;23:3287.

4. Wuebbens MM, Rajagopalan KV. Structural characterization of a molybdopterin precursor. J Biol Chem. 1993;268:13493-8.

Santamaria-Araujo JA, Fischer B, Otte T, et al. The

tetrahydropyranopterin structure of the sulfur-free and

metal-free molybdenum cofactor precursor. J Biol Chem. 2004:279:15994-9.

6. Reiss J, Cohen N, Dorche C, et al. Mutations in a polycistronic nuclear gene associated with molybdenum cofactor deficiency. Nat Genet. 1998:20:51-3.

7. Stallmeyer B, Drugeon G, Reiss J, et al. Human molybdopterin synthase gene: Identification of a bicistronic transcript with overlapping reading frames. Am J Hum Genet. 1999;64:698-705

8. Matthies A, Rajagopalan KV, Mendel RR, Leimkühler S. Evidence for the physiological role of a rhodanese-like protein for the biosynthesis of the molybdenum cofactor in humans. Proc Nat Acad Sci USA. 2004, 101:5946-51.

9. Llamas A, Mendel RR, Schwarz G. Synthesis of adenylated molybdopterin: An essential step for molybdenum insertion. Biol Chem. 2004;279:55241-6.

10. Rajagopalan $\mathrm{KV}$, Johnson JL. The pterin molybdenum cofactors J Biol Chem. 1992;267:10199-202.

11. Ataya FS, Witte CP, Galvan A, et al. Mcp1 encodes the molybdenum cofactor carrier protein in Chlamydomonas reinhardtii and participates in protection, binding, and storage functions of the cofactor. J Biol Chem. 2003;278:10885-90.

2. Klein JM, Schwarz G. Cofactor-dependent maturation of mammalian sulfite oxidase links two mitochondrial import pathways. J Cell Sci. 2012;125:4876-85.

13. Kaufholdt D, Gehl C, Geisler M, et al. Visualization and quantification of protein interactions in the biosynthetic pathway of molybdenum cofactor in Arabidopsis thaliana. J Exp Bot. 2013;64:2005-16

14. Bittner $F$, Oreb M, Mendel RR. ABA3 is a molybdenum cofactor sulfurase required for activation of aldehyde oxidase and xanthine dehydrogenase in Arabidopsis thaliana. J Biol Chem. 2001:276:40381-4.

15. Mudd SH, Irreverre F, Laster L. Sulfite oxidase deficiency in man: demonstration of the enzymatic defect. Science. 1967:156:1599-602.

16. Irreverre F, Mudd SH, Heizer WD, Laster L. Sulfite oxidase deficiency: Studies of a patient with mental retardation, dislocated ocular lenses, and abnormal urinary excretion of S-sulfo-l-cysteine, sulfite, and thiosulfate. Biochem Med. 1967; 1:187-217.

17. Duran $\mathrm{M}$, Beemer FA, van de Heiden $\mathrm{C}$, et al. Combined deficiency of xanthine oxidase and sulphite oxidase: a defect of molybdenum metabolism or transport? J Inherit Metab Dis. 1978:1:175-8

18. Johnson JL, Duran M. Molybdenum cofactor deficiency and isolated sulfite oxidase deficiency. In: Scriver C, Beaudet A, Sly $W$, Valle $D$ (eds). The metabolic and molecular bases of inherited disease. New York: MCGraw-Hill, 2001:3163-77.

19. Mechler K, Mountford WK, Hoffmann GF, Ries M. Ultra-orphan diseases: a quantitative analysis of the natural history of molybdenum cofactor deficiency. Genet Med. 2015;17:965-70.

20. Misko AL, Liang Y, Kohl JB, Eichler F. Delineating the phenotypic spectrum of sulfite oxidase and molybdenum cofactor deficiency. Neurol Genet. 2020;6:e486.

21. Spiegel R, Schwahn B, Scribner C L, Confer N. A natural history study of molybdenum cofactor (MOCO) and isolated sulfite oxidase deficiencies (ISOD). Poster 552. I Inherit Metab Dis. 2019;42 Suppl.:339.

22. Claerhout $H$, Witters $P$, Regal $L$, et al. Isolated sulfite oxidase deficiency. I Inherit Metab Dis. 2018;41:101-8.

23. Arican P, Gencpinar P, Kirbiyik $O$, et al. The clinical and molecula characteristics of molybdenum cofactor deficiency due to MOCS2 mutations. Pediatr Neurol. 2019:99:55-9.

24. Huijmans JGM, Schot R, de Klerk JBC, et al. Molybdenum Huljmans JGM, Schot R, de Klerk JBC, et al. Molybden
cofactor deficiency: Identification of a patient with homozygote mutation in the MOCS3 gene. Am J Med Genet A. 2017;173:1601-6

25. Tian Q, Cao Y, Shu L, et al. Case report: compound heterozygous variants in MOCS3 identified in a Chinese infant with molybdenum cofactor deficiency. Front Genet 2021;12:651878

26. Reiss J, Hahnewald R. Molybdenum cofactor deficiency: Mutations in GPHN, MOCS1, and MOCS2. Hum Mutat. 2011;32:10-8

27. Carmi-Nawi $\mathrm{N}$, Malinger $\mathrm{G}$, Mandel $\mathrm{H}$, et al. Prenatal brain disruption in molybdenum cofactor deficiency. I Child Neurol. 2011;26:460-4.
28. Lubout CMA, Derks TGJ, Meiners L, et al. Molybdenum cofactor deficiency type A: Prenatal monitoring using MRI. Eur J Paediatr Neurol. 2018;22:536-40.

29. Alonzo Martínez MC, Cazorla E, Cánovas E, et al. Molybdenum cofactor deficiency: Mega cisterna magna in two consecutive pregnancies and review of the literature. App/ Clin Genet. 2020;13:49-55.

30. Veldman A, Hennermann JB, Schwarz G, et al. Timing of cerebral developmental disruption in molybdenum cofactor deficiency. $J$ Child Neurol. 2011;26:1059-60; author reply 1061

31. Reiss J, Bonin M, Schwegler $\mathrm{H}$, et al. The pathogenesis of molybdenum cofactor deficiency, its delay by maternal molybdenum cofactor deficiency, its delay by maternal
clearance, and its expression pattern in microarray analysis. clearance, and its expression patten
Mol Genet Metab. 2005:85:12-20.

32. Karunakar P, Krishnamurthy $S$, Kasinathan $A$, et al. Renal stones in an infant with microcephaly and spastic quadriparesis: Answers. Pediatr Nephrol. 2020;35:987-9.

33. Mayr SJ, Sass JO, Vry J, et al. A mild case of molybdenum cofactor deficiency defines an alternative route of MOCS1 protein maturation. J Inherit Metab Dis. 2018;41:187-96.

34. Scelsa B, Gasperini S, Righini A, et al. Mild phenotype in molybdenum cofactor deficiency: A new patient and review of the literature. Mol Genet Genomic Med. 2019;7:e657.

35. Hinderhofer K, Mechler K, Hoffmann GF, et al. Critical appraisal of genotype assessment in molybdenum cofactor deficiency Inherit Metab Dis. 2017;40:801-11.

36. Veldman A, Santamaria-Araujo JA, Sollazzo S, et al. Successful treatment of molybdenum cofactor deficiency type A with treatment of molybdenum cofactor
CPMP. Pediatrics. 2010;125:e1249-54.

37. Schwarz G, Mendel RR, Ribbe MW. Molybdenum cofactors, enzymes and pathways. Nature. 2009;460:839-47.

38. Wyse ATS, Grings M, Wajner M, Leipnitz G. The role of oxidative stress and bioenergetic dysfunction in sulfite oxidase deficiency: Insights from animal models. Neurotox Res. 2019;35:484-94.

39. Zhang X, Vincent AS, Halliwell B, Wong KP. A mechanism of sulfite neurotoxicity: direct inhibition of glutamate dehydrogenase. J Biol Chem. 2004;279:43035-45.

40. Grings M, Moura AP, Amaral AU, et al. Sulfite disrupts brain mitochondrial energy homeostasis and induces mitochondrial permeability transition pore opening via thiol group modification. Biochim Biophys Acta. 2014;1842:1413-22.

41. Grings M, Seminotti B, Karunanidhi A, et al. ETHE1 and MOCS1 deficiencies: Disruption of mitochondrial bioenergetics, dynamics, redox homeostasis and endoplasmic reticulumdynamics, redox homeostasis and endoplasmic retics
mitochondria crosstalk in patient fibroblasts. Sci Rep. mitochondria

42. Mellis AT, Roeper J, Misko AL, et al. Sulfite alters the mitochondrial network in molybdenum cofactor deficiency. Front Genet. 2021;11:594828.

43. Patneau DK, Mayer ML. Structure-activity relationships for amino acid transmitter candidates acting at N-methyl-Daspartate and quisqualate receptors. J Neurosci. 1990;10:238599.

44. Nahum-Levy R, Lipinski D, Shavit S, Benveniste M. Desensitization of NMDA receptor channels is modulated by glutamate agonists. Biophys J. 2001;80:2152-66.

45. Olney JW, Misra CH, de Gubareff T. Cysteine-S-sulfate: brain damaging metabolite in sulfite oxidase deficiency. Neuropathol Exp Neurol 1975;34:167-77.

46. Kumar A, Dejanovic B, Hetsch F, et al. S-sulfocysteine/NMDA receptor-dependent signalling underlies neurodegeneration in molybdenum cofactor deficiency. J Clin Invest. 2017:127:436578.

47. Plate J, Sassen WA, Hassan AH, et al. S-Sulfocysteine induces seizure-like behaviors in zebrafish. Front Pharmacol. 2019;10:122

48. Footitt EJ, Heales SJ, Mills PB, et al. Pyridoxal 5'-phosphate in cerebrospinal fluid; factors affecting concentration. I Inherit Metab Dis. 2011;34:529-38.

49. Mills PB, Footitt EJ, Ceyhan $\mathrm{S}$, et al. Urinary AASA excretion is elevated in patients with molybdenum cofactor deficiency and isolated sulphite oxidase deficiency. I Inherit Metab Dis. 2012;35:1031-6.

50. Boles RG, Ment LR, Meyn MS, et al. Short-term response to dietary therapy in molybdenum cofactor deficiency. Ann Neurol. 1993;34:742-4.

51. Struys EA, Nota B, Bakkali A, et al. Pyridoxine-dependent epilepsy with elevated urinary $\alpha$-amino adipic semialdehyde in molybdenum cofactor deficiency. Pediatrics. 2012;130:e1716-9.

52. Johnson JL, Wuebbens MM, Mandell R, Shih VE. Molybdenum cofactor biosynthesis in humans. Identification of two complementation groups of cofactor-deficient patients and preliminary characterization of a diffusible molybdopterin precursor. J Clin Invest. 1989;83: 897-903.

53. Lee H-J, Adham IM, Schwarz G, et al. Molybdenum cofactordeficient mice resemble the phenotype of human patients Hum Mol Genet. 2002:11:3309-17.

54. Schwarz G, Santamaria-Araujo JA, Wolf S, et al. Rescue of lethal molybdenum cofactor deficiency by a biosynthetic precursor from Escherichia coli. Hum Mol Gen. 2004;13:1249-55. 55. NCT00957749. A Multicenter, Open-Label Study of the Safety, Tolerability, and Pharmacodynamics of Intravenously Administered cPMP (Precursor Z) in Patients With Molybdenum Cofactor Deficiency Type A. https://clinicaltrials.gov/ct2/show/ NCT00957749? cond=Molybdenum+Cofactor+Deficiency \&draw $=1 \&$ rank $=3$

56. Schwahn BC, Galloway PG, Bowhay S, et al. Successful treatment of two neonates with molybdenum cofactor deficiency (MOCD) type a, using cyclic pyranopterin monophosphate (CPMP). I Inherit Metab Dis. 2010;33:S29.

57. Schwahn BC, Galloway P, Bowhay S. Follow-up of two infants with molybdenum cofactor deficiency (MOCD) group A, on with molybdenum cofactor deficiency (MOCD) group A, on long-term treatment with cyclic pyranopterin mon
(CPMP). I Inherit Metab Dis. 2011;34 Suppl. 3:S84.

58. Hitzert MM, Bos AF, Bergman KA, et al. Favorable outcome in a newborn with molybdenum cofactor type A deficiency treated with cPMP. Pediatrics. 2012;130:e1005-10

59. Schwahn BC, Van Spronsen FJ, Belaidi AA, et al. Efficacy and safety of cyclic pyranopterin monophosphate substitution in severe molybdenum cofactor deficiency type $\mathrm{A}$ : a prospective cohort study. Lancet. 2015;386:1955-63.

60. Public summary of opinion on orphan designation: Cyclic pyranopterin monophosphate for the treatment of molybdenum cofactor deficiency type A. 2015. Available at: https://www.ema.europa.eu/en/documents/orphanat: https://Www.ema.europa.eu/en/documents/orphandesignation/eu/3/10/777-public-summary-opinion-orphan-
designation-cyclic-pyranopterin-monophosphate-treatmentdesignation-cyclic-pyranopterin-mono
molybdenum en pdf (accessed XXX)

61. Clinch K, Watt DK, Dixon RA, et al. Synthesis of cyclic pyranopterin monophosphate, a biosynthetic intermediate in the molybdenum cofactor pathway. J Med Chem. 2013;56:1730-8.

62. BridgeBio Pharma And Affiliate Origin Biosciences Announce FDA Approval Of NULIBRYTM (Fosdenopterin), The First And Only Approved Therapy To Reduce The Risk Of Mortality In Patients With MoCD Type A. 2021. Available at: https:// bridgebio.com/news/bridgebio-pharma-and-affiliate-originbiosciences-announce-fda-approval-of-nulibry-fosdenopterinthe-first-and-only-approved-therapy-to-reduce-the-risk-ofmortality-in-patients-with-mocd-t (accessed XXX).

63. NCT01640717. A Retrospective, Observational, Noninterventional Data Collection Study for Patients With Molybdenum Cofactor Deficiency Who Have Been Previously Treated With Cyclic Pyranopterin Been Previously Treated With Cyclic Pyranopterin
Monophosphate (CPMP). https://clinicaltrials.gov/ct2/show/ NCT01640717? cond=Molybdenum+Cofactor+Deficiency $\& d r a w=1 \&$ rank $=2$ (accessed 7 December 2021)

64. NCT01894165. A Phase 1, Randomized, Blinded, PlaceboControlled, Single-Dose, Sequential-Cohort, Dose-Escalation Study to Evaluate the Safety, Tolerability and Pharmacokinetics of ALXN1101 in Healthy Adult Subjects. https://clinicaltrials.gov/ ct2/show/NCT01894165? cond=Molybdenum+Cofactor+ Deficiency\&draw=1\&rank=6 (accessed 7 December 2021).

65. NCT02047461. A Phase 2, Multicenter, Multinational, OpenLabel, Dose-Escalation Study to Evaluate the Safety and Efficacy of ORGNO01 (Formerly ALXN1101) in Pediatric Patients With Molybdenum Cofactor Deficiency (MoCD) Type A Currently Treated With Recombinant Escherichia Coli-derived Cyclic Pyranopterin Monophosphate (rcPMP). https://clinicaltrials.gov/ Pyranopterin Monophosphate (rcPMP). https://clinicaltrials
ct2/show/NCT02047461? cond=Molybdenum+Cofactor+ Deficiency\&draw=1\&rank=4 (accessed 7 December 2021). Deficiency\&draw $=1 \&$ rank $=4$ (accessed 7 December 2021).
NCT02629393. A Phase 2/3, Multicenter, Multinational, NCT02629393. A Phase 2/3, Multicenter, Multinational,
Open Label Study to Evaluate the Efficacy and Safety of ORGNO01 (Formerly ALXN1101) in Neonates, Infants and Children With Molybdenum Cofactor Deficiency (MOCD) Type A. https://clinicaltrials.gov/ct2/show/ NCT02629393? cond=Molybdenum+Cofactor+ Deficiency\&draw=2\&rank=1 (accessed 7 December 2021).

67. Nulibry ${ }^{\mathrm{TM}}$ Prescribing Information. Available at: www.nulibry. com/pdfs/nulibry-prescribing-information-v2.pdf (accessed 18 July 2021).

68. Watsky E, Kuklin N, Hamilton M, Barr S. Maintenance of diseaserelevant biomarker improvement in patients with Molybdenum Cofactor Deficiency (MoCD) Type A administered ALXN1101, a synthetic form of cyclic pyranopterin monophosphate. JPN a synthetic form of Cyclic
2017:21 Suppl. 1:E124.

69. Novotny JA, Peterson CA. Molybdenum. Adv Nutr. 2018;9:272-3.

. Domellöf M, Szitanyi P, Simchowitz V, et al. ESPGHAN/ESPEN/ ESPR/CSPEN guidelines on pediatric parenteral nutrition: Iro and trace minerals. Clin Nutr. 2018;37:2354-9.

71. Stipanuk MH, Dominy JE Jr, Lee Jl, Coloso RM. Mammalian cysteine metabolism: new insights into regulation of cysteine metabolism. J Nutr. 2006;136 Suppl.:1652S-9S.

72. National Center for Biotechnology Information. Pubchem Compound Summary for CID 135894389 2D Structure. https:// pubchem.ncbi.nlm.nih.gov/compound/135894389. (Accessed 7 December 2021). 